\title{
A renormalized Gross-Pitaevskii Theory and vortices in a strongly interacting Bose gas
}

\author{
Ch. Moseley \\ E-mail: Christopher.Moseley@Physik.Uni-Augsburg.de \\ Institut für Physik, Universität Ausgburg, D-86135 Augsburg, Germany \\ K. Ziegler \\ Institut für Physik, Universität Ausgburg, D-86135 Augsburg, Germany
}

\begin{abstract}
We consider a strongly interacting Bose-Einstein condensate in a spherical harmonic trap. The system is treated by applying a slave-boson representation for hard-core bosons. A renormalized Gross-Pitaevskii theory is derived for the condensate wave function that describes the dilute regime (like the conventional Gross-Pitaevskii theory) as well as the dense regime. We calculate the condensate density of a rotating condensate for both the vortex-free condensate and the condensate with a single vortex and determine the critical angular velocity for the formation of a stable vortex in a rotating trap.
\end{abstract}

PACS numbers: 03.75.Hh,05.30.Jp,32.80.Pj 
A renormalized Gross-Pitaevskii Theory and vortices in a strongly interacting Bose gas2

\section{Introduction}

In this paper we shall study two aspects of a strongly interacting Bose gas at high density. One is related to a consistent treatment of a strongly interacting Bose gas in terms of an effective Gross-Pitaevskii (GP) Eq. with renormalized parameters. The second aspect is related to the formation of a vortex in a trapped condensate in the presence of strong interaction.

The stationary form of the conventional GP Eq.

$$
\left[-\frac{\hbar^{2}}{2 m} \nabla^{2}-\mu+V(\mathbf{r})+g|\Phi(\mathbf{r})|^{2}\right] \Phi(\mathbf{r})=0
$$

describes the condensate order parameter $\Phi$ of a Bose gas in a trapping potential at zero temperature, where $\mu$ is the chemical potential and $g$ the repulsive coupling constant [1, 2, 3]. In the absence of a trapping potential, a solution of Eq. (1) is given by

$$
|\Phi|^{2}=\frac{\mu}{g}
$$

This describes a linearly increasing condensate density (the latter is proportional to $|\Phi|^{2}$ ) with respect to the chemical potential. Although it takes the repulsion into account by a factor $1 / g$ which is decreasing with increasing coupling constant $g$, the saturation of $n_{0}$ cannot be seen in this solution. From the physical point of view, in a realistic description for large densities, the particle density must saturate because there is a finite scattering volume around each particle. Furthermore, for increasing particle density, the condensate density should reach a maximum and for even larger densities, decrease again until its total destruction, because of the increasing interparticle interaction. This behaviour has also been found by variational perturbation theory [4] and diffusion Monte Carlo calculations [5]. In other words, the strong effect of the repulsion in a dense condensate is not really described by the conventional GP Eq. In order to describe condensates at higher densities, the second order term in the low-density expansion of the energy density has been taken into account which lead to a modified GP theory [1, 5, 6, 7].

Although in many experimentally realized situations the BEC is in the weakly interacting regime where it is well described by GP theory, it might be possible to reach the strongly interacting regime. The main problem at high particle densities is the instability of the Bose gas by the formation of molecules due to three-particle interactions [8]. Here, we will assume that molecule formation does not occur. This might be unrealistic for some systems, but in others it is not, e.g. for Bose gases in optical lattices.

It has been shown that the slave-boson approach to a hard-core Bose gas provides a mean-field Eq., similar to the GP Eq., that leads to a saturation of the condensate $n_{0} \leq 1$ [9, 10, 11, 12, 13]. Here we will discuss a simplified version of this mean-field equation which is the same type of nonlinear Schrödinger Eq. like in the GP approach. However, in contrast to the latter the parameters are renormalized such that Eq. (2) 
becomes

$$
\left|\Phi_{\mathrm{R}}\right|^{2}=\frac{\mu_{\mathrm{R}}}{g_{\mathrm{R}}},
$$

where the renormalized parameters $\mu_{\mathrm{R}}$ and $g_{\mathrm{R}}$ are functions of the bare parameters $\mu$ and $n_{0}$. At the phase transition between the non-condensed Bose gas and the BEC $\mu_{\mathrm{R}}$ vanishes, then increases linearly with increasing $\mu$, reaches a maximum and decreases again until the condensate is destroyed totally due to strong interaction effects. We use this approach to calculate the condensate density profile of a trapped BEC.

The formation of vortices in a rotating condensate is understood as a local destruction of the condensate. From the depletion effect due to strong interaction it can be anticipated that the tendency of vortex formation is enhanced in a dense Bose gas. This implies a reduction of the critical angular velocity $\Omega_{c}$ with increasing density by the interaction. Vortex formation in rotating traps in the region beyond the validity of GP has also been studied by the modified GP theory and by variational Monte Carlo methods [7].

The paper is organized as follows: In the following section we survey the results of the slave-boson approach for a hard-core Bose gas on a lattice and derive a corresponding mean field theory for a continuous system in an external trap potential. Then we derive the renormalized GP Eq. and evaluate solutions with and without a straight vortex and determine the critical angular velocity for the vortex formation.

\section{The Model}

The slave-boson representation of a strongly interacting Bose gas is based on the idea that the bosons fill the space with finite density, where each particle occupies a lattice cell related to a sphere with radius $a_{s}$ (s-wave scattering length) [10]. Singly occupied and empty sites are described by two complex fields $b_{x}$ and $e_{x}$, respectively [11]. Here, $b_{x}^{*}$ and $b_{x}$ represent a creation and annihilation process of a boson at site $x$, while $e_{x}^{*}$ and $e_{x}$ represent the creation and annililation of a "hole". In the functional integral representation [14], the grand canonical partition function in classical approximation is given as

$$
\begin{aligned}
Z=\int \exp [- & \left.\beta \sum_{x, x^{\prime}} b_{x}^{*} e_{x} t_{x, x^{\prime}} e_{x^{\prime}}^{*} b_{x^{\prime}}+\beta \sum_{x} \mu_{x}\left|b_{x}\right|^{2}\right] \\
& \times \prod_{x} \delta\left(\left|e_{x}\right|^{2}+\left|b_{x}\right|^{2}-1\right) \mathrm{d} b_{x} \mathrm{~d} b_{x}^{*} \mathrm{~d} e_{x} \mathrm{~d} e_{x}^{*},
\end{aligned}
$$

where $1 / \beta=k_{\mathrm{B}} T$ is the thermal energy. In a $d$-dimensional lattice,

$$
\hat{t}_{x, x^{\prime}}= \begin{cases}-J /(2 d) & \text { if } x, x^{\prime} \text { nearest neighbours } \\ 0 & \text { else }\end{cases}
$$

is the nearest-neighbour tunneling rate and $\mu_{x}=\mu-V_{x}$ is the space-dependent chemical potential that includes the trapping potential $V_{x}$. Instead of $\mu_{x}$ we will write only $\mu$ subsequently and assume implicitly that the effective chemical potential can depend on 
space. The fields $e$ and $b$ are dimensionless. Moreover, we rescale all physical energies by a multiple of the hopping rate $\alpha J$ to obtain dimensionless quantities:

$$
\hat{t} \rightarrow \hat{t}^{\prime}=\frac{1}{\alpha J} \hat{t}, \quad \mu \rightarrow \mu^{\prime}=\frac{1}{\alpha J} \mu, \quad \beta \rightarrow \beta^{\prime}=\alpha J \beta .
$$

The hopping term is quartic in the field variables, due to the fact that a hopping process is characterized as an "exchange process" of a boson and an empty site. The $\delta$ function enforces the constraint that each lattice site is either singly occupied or empty but excludes a multiple occupation.

A similar approach has been applied to the Bose-Hubbard model which allows multiple occupation, by introducing additional fields, one for each occupation number [12, 13]. The well-known zero-temperature phase diagram with Mott-insulating phases for integer lattice fillings $n=0,1,2, \ldots$ ("lobes") was found. In contrary, our hard-core Boson model is restricted to the two lattice fillings $n=0,1$ which is a simplification, but contains all relevant aspects of Bose-Einstein condensation with repulsive interaction. A second simplification of our approach is, that we neglect quantum fluctuations by treating the grand-canonical partition function in classical approximation. This allows us to integrate out the constraint exactly. On the other hand, it restricts the applicability of our approach to non-zero temperatures.

Two new fields are introduced by a Hubbard-Stratonovich transformation, the complex field $\Phi_{x}$ which describes the condensate wave function, and the real field $\varphi_{x}$ which is related to the total density of bosons [10]:

$$
\begin{aligned}
Z=\int \exp \{ & -\beta^{\prime}\left[\sum_{x, x^{\prime}} \Phi_{x}^{*}\left(\mathbf{1}-\hat{t}^{\prime}\right)_{x, x^{\prime}}^{-1} \Phi_{x^{\prime}}+\sum_{x} \varphi_{x}^{2}\right. \\
& \left.\left.+\sum_{x}\left(e_{x}, b_{x}\right)\left(\begin{array}{cc}
2 \varphi_{x}+1 & \Phi_{x} \\
\Phi_{x}^{*} & -\mu^{\prime}
\end{array}\right)\left(\begin{array}{c}
e_{x}^{*} \\
b_{x}^{*}
\end{array}\right)\right]\right\} \\
& \times \prod_{x} \delta\left(\left|b_{x}\right|^{2}+\left|e_{x}\right|^{2}-1\right) \mathrm{d} b_{x} \mathrm{~d} b_{x}^{*} \mathrm{~d} e_{x} \mathrm{~d} e_{x}^{*} \mathrm{~d} \Phi_{x} \mathrm{~d} \Phi_{x}^{*} \mathrm{~d} \varphi_{x} .
\end{aligned}
$$

Integration over the fields $\Phi$ and $\varphi$ leads back to Eq. (3). On the other hand, the fields $b_{x}$ and $e_{x}$ can be integrated out in (5) because they appear in the exponent as quadratic forms. This leads to the partition function

$$
Z=\int e^{-S_{b}-S_{1}} \prod_{x} \mathrm{~d} \Phi_{x} \mathrm{~d} \Phi_{x}^{*}
$$

with the kinetic part of the action

$$
S_{b}=\beta^{\prime} \sum_{x, x^{\prime}} \Phi_{x}\left(\mathbf{1}-\hat{t}^{\prime}\right)_{x, x^{\prime}}^{-1} \Phi_{x^{\prime}}^{*}
$$

and the potential part

$$
S_{1}=-\sum_{x} \log \left(\int_{-\infty}^{\infty} e^{-\beta^{\prime} \varphi_{x}^{2}} \frac{\sinh \left[\beta^{\prime} \sqrt{\left(\varphi_{x}+\frac{\mu^{\prime}}{2}\right)^{2}+\left|\Phi_{x}\right|^{2}}\right]}{\beta^{\prime} \sqrt{\left(\varphi_{x}+\frac{\mu^{\prime}}{2}\right)^{2}+\left|\Phi_{x}\right|^{2}}} \mathrm{~d} \varphi_{x}\right):=-\sum_{x} Z_{x} .
$$


A renormalized Gross-Pitaevskii Theory and vortices in a strongly interacting Bose gas5

The condensate density can be identified with

$$
n_{0}=\frac{\left|\Phi_{x}\right|^{2}}{(1+1 / \alpha)^{2}}
$$

like argued in Appendix A, and the total particle density is given by the expectation value [10]

$$
n_{\mathrm{tot}}=\left\langle\varphi_{x}\right\rangle+\frac{1}{2}
$$

We apply a saddle-point approximation to the integration in Eq. (6). This is controlled by the minimized action, which means that we have to solve the equation

$$
\frac{\partial S}{\partial \Phi_{x}^{*}}=0
$$

This yields the mean-field equation

$$
\beta^{\prime} \sum_{x^{\prime}}\left(\mathbf{1}-\hat{t}^{\prime}\right)_{x, x^{\prime}}^{-1} \Phi_{x^{\prime}}-\left[\frac{\partial}{\partial\left(\left|\Phi_{x}\right|^{2}\right)} \log Z_{x}\right] \Phi_{x}=0 .
$$

In order to derive a mean-field equation which is applicable to a continuous trapping potential, we perform the continuum approximation of (11). If the field $\Phi_{\mathbf{r}}$ is varying only very slowly between neighbouring lattice sites, we can approximate

$$
\left(\mathbf{1}-\hat{t}^{\prime}\right)_{x, x^{\prime}}^{-1} \approx \frac{1}{1+1 / \alpha}\left(\delta_{x, x^{\prime}}+\frac{1}{1+1 / \alpha} \alpha J\left(J \delta_{x, x^{\prime}}+\hat{t}_{x, x^{\prime}}\right)\right),
$$

and perform the substitution

$$
\sum_{x^{\prime}}\left(J \delta_{x, x^{\prime}}+\hat{t}_{x, x^{\prime}}\right) \rightarrow-J a^{2} \nabla^{2} .
$$

This leads to the equation

$$
\left[-\frac{J a^{2}}{6} \nabla^{2}+(1+\alpha) J-\frac{(1+1 / \alpha)^{2}}{\beta} \frac{\partial}{\partial\left(|\Phi(\mathbf{r})|^{2}\right)} \log Z(\mathbf{r})\right] \Phi(\mathbf{r})=0 .
$$

with

$$
Z(\mathbf{r})=\int e^{-\beta^{\prime} \varphi_{\mathbf{r}}^{2}} \frac{\sinh \left[\beta^{\prime} \sqrt{\left(\varphi_{\mathbf{r}}+\mu^{\prime} / 2\right)^{2}+|\Phi(\mathbf{r})|^{2}}\right]}{\left[\beta^{\prime} \sqrt{\left(\varphi_{\mathbf{r}}+\mu^{\prime} / 2\right)^{2}+|\Phi(\mathbf{r})|^{2}}\right]} \mathrm{d} \varphi_{\mathbf{r}}
$$

for the spacially dependent order parameter $\Phi(\mathbf{r})$ in a three-dimensional space and $a$ is the lattice constant of the discrete system. In the continuum a looses its identity as lattice constant, but describes a characteristic length scale in Eq. (12), and can be interpreted as the spacial extension of a boson. Thus, it should be of the same order of magnitude as the $s$-wave scattering length $a_{s}$ [1]. Eq. (12) is the analogue of the GP Eq. in the case of our slave-boson approach. The parameters can be identified with those of the conventional GP Eq.: The mass $m$ of the particles is given by the hopping constant $J$ and the lattice constant $a$ via

$$
\frac{\hbar^{2}}{2 m} \equiv \frac{J a^{2}}{6} \text {. }
$$


A renormalized Gross-Pitaevskii Theory and vortices in a strongly interacting Bose gas6

\section{Renormalized Gross-Pitaevskii Equation}

The continuum limit of the action defined by Eq. (7) and (8) is

$$
S=\int\left\{\beta^{\prime} \Phi^{*}(\mathbf{r})\left[-\frac{\alpha}{(1+\alpha)^{2}} \frac{a^{2}}{6} \nabla^{2}+\frac{1}{1+1 / \alpha}\right] \Phi(\mathbf{r})-\log Z(\mathbf{r})\right\} \mathrm{d}^{d} r
$$

Applying the variational principle

$$
\frac{\partial S}{\partial \Phi_{x}^{*}}=0
$$

we obtain the full mean-field Eq. (12) directly. If the order parameter $\Phi$ is small, we can expand the potential part of the action up to fourth order:

$$
\frac{1}{1+1 / \alpha}|\Phi|^{2}-\frac{1}{\beta^{\prime}} \log Z(\mathbf{r})=a_{0}\left(\mu^{\prime}\right)+a_{2}\left(\mu^{\prime}\right)|\Phi|^{2}+\frac{1}{2} a_{4}\left(\mu^{\prime}\right)|\Phi|^{4}+\mathcal{O}\left(|\Phi|^{6}\right),
$$

where we have introduced the coefficients

$$
\begin{aligned}
& a_{0}\left(\mu^{\prime}\right)=-\left.\frac{1}{\beta^{\prime}} \log Z(\mathbf{r})\right|_{\Phi=0} \\
& a_{2}\left(\mu^{\prime}\right)=-\left.\frac{1}{\beta^{\prime}} \frac{\partial}{\partial|\Phi|^{2}} \log Z(\mathbf{r})\right|_{\Phi=0}+\frac{1}{1+1 / \alpha} \\
& a_{4}\left(\mu^{\prime}\right)=-\left.\frac{1}{\beta^{\prime}} \frac{\partial^{2}}{\left(\partial|\Phi|^{2}\right)^{2}} \log Z(\mathbf{r})\right|_{\Phi=0} .
\end{aligned}
$$

Further, we introduce the rescaled field

$$
\Phi_{\mathrm{R}}(\mathbf{r})=\frac{1}{1+1 / \alpha} \Phi(\mathbf{r})
$$

which we identify with the condensate wave function of our renormalized GP theory. We now introduce the renormalized coefficients

$$
\mu_{\mathrm{R}}(\mu, J) \equiv-\frac{(1+\alpha)^{2}}{\alpha} J a_{2}\left(\mu^{\prime}\right)
$$

and

$$
g_{\mathrm{R}}(\mu, J) \equiv \frac{(1+\alpha)^{4}}{\alpha^{3}} J a_{4}\left(\mu^{\prime}\right) .
$$

After neglecting the term of order $|\Phi|^{6}$ in the expansion (15), we get the renormalized Gross-Pitaevskii (RGP) Eq.

$$
\left[-\frac{J a^{2}}{6} \nabla^{2}-\mu_{\mathrm{R}}(\mu, J)+g_{\mathrm{R}}(\mu, J)\left|\Phi_{\mathrm{R}}(\mathbf{r})\right|^{2}\right] \Phi_{\mathrm{R}}(\mathbf{r})=0 .
$$

It has the same form as the conventional GP Eq. (1), when $\mu$ and $g$ are replaced by $\mu_{R}$ and $g_{R}$. Their $\mu$-dependence is plotted in Fig. 1. In the case of a trapping potential, where the chemical potential $\mu$ is space-dependent, $\mu_{\mathrm{R}}$ and $g_{\mathrm{R}}$ are space-dependent aswell. While $g_{\mathrm{R}}$ is always positive, $\mu_{\mathrm{R}}$ can change sign. A BEC exists if $\mu_{\mathrm{R}}>0$. The phase transition between the BEC and the non-condensate phase, i.e. the point at which the condensate order parameter vanishes, is given by the relation $\mu_{\mathrm{R}}(\mu, J)=0$. 
A renormalized Gross-Pitaevskii Theory and vortices in a strongly interacting Bose gas7

\section{Results}

\subsection{Zero-temperature result}

In the zero-temperature limit we can integrate out the $\varphi$-field in Eq. (8) exactly by a saddle-point integration, as shall be shown in this paragraph. Therefore, we write

$$
Z(\mathbf{r})=\frac{1}{2 \beta^{\prime}}\left(Z_{-}-Z_{+}\right)
$$

where

$$
Z_{ \pm}=\int_{-\infty}^{\infty} \frac{e^{-\beta^{\prime} f_{ \pm}\left(\varphi,|\Phi|^{2}\right)}}{\sqrt{\left(\varphi+\frac{\mu^{\prime}}{2}\right)^{2}+|\Phi|^{2}}} \mathrm{~d} \varphi
$$

and

$$
f_{ \pm}\left(\varphi,|\Phi|^{2}\right)=\varphi^{2} \pm \sqrt{\left(\varphi+\frac{\mu^{\prime}}{2}\right)^{2}+|\Phi|^{2}}
$$

It is possible to perform a saddle-point approximation by expanding the functions $f_{ \pm}$ up to second order in $\varphi$ around their minimum $\varphi_{0}$ :

$$
f_{ \pm}\left(\varphi,|\Phi|^{2}\right)=f_{ \pm}\left(\varphi_{0},|\Phi|^{2}\right)+\frac{1}{2} \frac{\partial^{2} f_{ \pm}}{\partial \varphi^{2}}\left(\varphi_{0},|\Phi|^{2}\right)\left(\varphi-\varphi_{0}\right)^{2}+\mathcal{O}\left(\varphi^{2}\right) .
$$

In the limit of large $\beta$, the saddle-point integration becomes exact and yields

$$
Z_{ \pm}=\sqrt{\frac{\pi}{\left(\varphi_{0}+\frac{\mu^{\prime}}{2}\right)^{2}+|\Phi|^{2}}} \frac{e^{-\beta^{\prime} f_{ \pm}\left(\varphi_{0},|\Phi|^{2}\right)}}{\sqrt{\frac{\beta^{\prime}}{2} \frac{\partial^{2} f_{ \pm}\left(\varphi_{0},|\Phi|^{2}\right)}{\partial \varphi^{2}}}} .
$$

The minimum is found to satisfy the equation

$$
|\Phi|^{2}=\left(\varphi_{0}+\frac{\mu^{\prime}}{2}\right)^{2}\left(\frac{1}{4 \varphi_{0}^{2}}-1\right)
$$

and we have

$$
f_{ \pm}\left(\varphi_{0}\right)=\varphi_{0}^{2}-\frac{1}{2}-\frac{\mu^{\prime}}{4 \varphi_{0}} ; \frac{\partial^{2} f_{ \pm}\left(\varphi_{0}\right)}{\partial \varphi^{2}}=2-\frac{8|\Phi|^{2} \varphi_{0}^{3}}{\left(\varphi_{0}+\frac{\mu^{\prime}}{2}\right)^{3}}
$$

We find the following zero-temperature result for a translational invariant condensate ( $\Phi_{x} \equiv \Phi=$ const) from the mean-field Eq. (12) : For the condensate density we find

$$
n_{0}=\frac{|\Phi|^{2}}{(1+1 / \alpha)^{2}}= \begin{cases}\frac{1}{4}\left(1-\frac{\mu^{2}}{J^{2}}\right) & \text { if }-J<\mu<J \\ 0 & \text { else },\end{cases}
$$

and the total particle density given by Eq. (10) is

$$
n_{\text {tot }}=\varphi_{0}+\frac{1}{2}= \begin{cases}0 & \text { if } \mu \leq-J \\ \frac{1}{2}\left(1-\frac{\mu}{J}\right) & \text { if }-J<\mu<J \\ 1 & \text { if } J \leq \mu\end{cases}
$$

The solution of the mean-field equation is plotted in Fig. 2 for zero-temperature and near the critical temperature $T_{c}$ where the BEC breaks down. In the dilute regime at $T=0$, the chemical potential can be written as $\mu=-J+\Delta \mu$, where $\Delta \mu \ll J$. In this limiting case we find $\mu_{\mathrm{R}}=\Delta \mu+\mathcal{O}\left(\Delta \mu^{2}\right)$ and $g_{\mathrm{R}}=2 J$, which is consistent with the conventional GP Eq. with a shifted chemical potential. 


\subsection{Vortex-free trapped condensate}

Assuming a dense condensate, where the repulsive interaction between bosons dominates their kinetic energy, we neglect the differential term in Eqs. (12) and (14). This is called the Thomas-Fermi (TF) approximation [1]. In the following we use a spherical trapping potential

$$
V(\mathbf{r})=\frac{m}{2} \omega_{\mathrm{ho}}^{2} \mathbf{r}^{2} .
$$

In typical experiments, the oscillator length $d_{\mathrm{ho}}=\sqrt{\hbar / m \omega_{\mathrm{ho}}}$ is of the order of a few $\mu \mathrm{m}$ [1], where $\omega_{\text {ho }}$ is the trap frequency measured in Hz. Considering, for instance, ${ }^{85} \mathrm{Rb}$ atoms near a Feshbach resonance [15], we can study a Bose gas in a dense regime with a scattering length $a_{s} \sim a \sim 200 \mathrm{~nm}$. In our calculations we choose the parameters

$$
\beta^{\prime}=1, \quad \frac{k_{\mathrm{B}} T}{\hbar \omega_{\mathrm{ho}}}=36.93, \quad \frac{a}{d_{\mathrm{ho}}}=0.1215,
$$

and keep the hopping constant $J$ fixed. Thus all energies can be scaled with $J$.

To calculate the profile of the condensate density in a BEC without vortex, we solve the TF equation

$$
(1+1 / \alpha)-(1+1 / \alpha)^{2} \frac{\partial}{\partial|\Phi(\mathbf{r})|^{2}} \log Z(\mathbf{r})=0 .
$$

In the RGP approximation (19), the solution is

$$
\left|\Phi_{\mathrm{R}}(\mathbf{r})\right|^{2}=\frac{\mu_{\mathrm{R}}}{g_{\mathrm{R}}}=-\frac{a_{2}\left(\mu^{\prime}\right)}{(1+1 / \alpha)^{2} a_{4}\left(\mu^{\prime}\right)} .
$$

Solutions for typical values of the parameters are plotted in Fig. 3, The results we get from the renormalised GP approximation shows only small deviations from the numerical solutions of Eq. (24). We find a condensate depletion at the trap center for $\mu^{\prime}=1$. This is due to the fact that the condensate is partly suppressed by strong interaction effects [4, 5, 11]. For $\mu^{\prime}=2$ the condensate is completely destroyed at the trap center. We find particle numbers in the condensate of the order $N_{0} \approx 10^{4} \ldots 10^{5}$.

We note that the total particle density $n_{\text {tot }}$ is much larger than the condensate density $n_{0}$ and takes values of about $0.5 a^{-3}$ at the trap center. Thus the interaction between the non-condensed and the condensed part of the Bose gas plays a significant role. This implies that the conventional GP Equation, which neglects the non-condensed part, is not reliable in this parameter regime.

\subsection{Rotating condensate with a single vortex}

In the case of a trap rotating about the $z$-axis with an angular velocity $\Omega$, one must include the additional angular momentum term $-\Omega L_{z} \Phi(\mathbf{r})$ to the left hand side of the differential equation (12), where $L_{z}$ is the $z$-component of the angular momentum operator. This term must also be kept in the TF approximation. The condensate wave function may develop a vortex then. We assume here a straight single vortex along the $z$-axis. This can be described by using cylindrical coordinates and the ansatz 
$\Phi(\mathbf{r})=\phi\left(r_{\perp}, z\right) e^{i \varphi}$, where $r_{\perp}$ is the distance from the $z$-axis and $\varphi$ the polar angle. The angular momentum operator is given as $L_{z}=-i \frac{\partial}{\partial \varphi}$. This gives rise to an additional term $\left(\Omega / \alpha J-a^{2} / \alpha\left(6 r_{\perp}^{2}\right)\right)|\Phi(\mathbf{r})|^{2} /(1+1 / \alpha)^{2}$ in the action (14). Instead of Eq. (24) we have to solve

$$
(1+1 / \alpha)+\left(\frac{a^{2}}{\alpha 6 r_{\perp}^{2}}-\frac{\Omega}{\alpha J}\right)-(1+1 / \alpha)^{2} \frac{\partial}{\partial|\Phi(\mathbf{r})|^{2}} \log Z(\mathbf{r})=0,
$$

and the solution in the RGP approximation is

$$
\left|\Phi_{\mathrm{R}}(\mathbf{r})\right|^{2}=-\frac{1}{(1+1 / \alpha)^{4} a_{4}\left(\mu^{\prime}\right)}\left[\left(\frac{a^{2}}{\alpha 6 r_{\perp}^{2}}-\frac{\Omega}{\alpha J}\right)+(1+1 / \alpha)^{2} a_{2}\left(\mu^{\prime}\right)\right] .
$$

A condensate that is rotating with given angular velocity $\Omega$ forms a stable vortex, if its total energy is lower than that of a vortex free condensate. This is equivalent to the condition

$$
S^{\mathrm{vort}}(\Omega)-S<0
$$

which can be checked numerically by using the TF approximation of Eq. (14) for a condensate with vortex $S_{\mathrm{FT}}^{\mathrm{vort}}(\Omega)$ and without vortex $S_{\mathrm{TF}}$. The critical angular velocity $\Omega_{\mathrm{c}}$ above which the vortex is stable is plotted against the number of condensed bosons $N_{0}$ in Fig. 4, where $N_{0}$ is given as

$$
N_{0}=\int \frac{1}{a^{3}}\left|\Phi_{\mathrm{R}}(\mathbf{r})\right|^{2} \mathrm{~d}^{3} \mathbf{r} .
$$

The RGP approximation is in good agreement with the results of the full mean-field equation. The decreasing critical angular velocity for higher values of $N_{0}$ indicates that a high interaction energy favours the formation of a vortex. It agrees with results derived from the GP Eq. by perturbation theory [16] as well as numerically [17].

Typical solutions for shapes of condensate density profiles of BECs with a stable single vortex are shown in Fig. 5. In contrast to the case without a vortex, the condensate is always completely destroyed at the trap center, a feature that also shows up in the conventional GP approximation [1]. Again, the RGP approximation is in good agreement with the numerical results from (26).

\section{Conclusion}

The slave-boson approach allowed us to study the condensation of a trapped highdensity Bose gas in a regime where the conventional Gross-Pitaevskii approach is not valid. Starting from the saddle-point approximation, we have derived a renormalized Gross-Pitaevskii Eq. with a space dependent coupling constant. This provides good results in comparison with the more complicated slave-boson saddle-point calculations. At high densities, we have found a depletion of the condensate at the trap center due to the interaction between the condensate and the non-condensate part of the Bose gas. This feature is not covered by the conventional Gross-Pitaevskii Eq. The behaviour of the critical angular velocity for the formation of a single vortex agrees qualitatively with previous results in the literature but supports also the formation of a vortex for increasing $N_{0}$. 
A renormalized Gross-Pitaevskii Theory and vortices in a strongly interacting Bose gas10

\section{Acknowledgements}

The authors want to thank O. Fialko, A. Gammal and K.K. Rajagopal for useful discussions.

[1] F. Dalfovo, S. Giorgini, L. P. Pitaevskii, and S. Stringari, Rev. Mod. Phys. 71, 463 (1999)

[2] L. Pitaevskii, and S. Stringari, Bose-Einstein Condensation (Clarendon, Oxford, 2003)

[3] A.J. Leggett, Rev. Mod. Phys. 73, 307(2001)

[4] H. Kleinert, S. Schmidt, and A. Pelster, Phys. Rev. Lett. 93, 160402 (2004)

[5] J. L. DuBois, and H. R. Glyde, Phys. Rev. A 68, 033602 (2003)

[6] A. Fabrocini, and A. Polls, Phys. Rev. A 60, 2319 (1999)

[7] J. K. Nilsen, J. Mur-Petit, M. Guilleumas, M. Hjorth-Jensen, and A. Polls, Phys. Rev. A 71, $053610(2005)$

[8] F. Kh. Abdullaev, A. Gammal, L. Tomio, and T. Frederico, Phys. Rev. A 63, 043604 (2001)

[9] G. Kotliar, and A. E. Ruckenstein, Phys. Rev. Lett. 57, 1362 (1986)

[10] K. Ziegler, Phys. Rev. A 62, 023611 (2000)

[11] K. Ziegler, Europhys. Lett. 23, 463 (1993); K. Ziegler, and A. Shukla, Phys. Rev. A 56, 1438 (1997)

[12] D. B. M. Dickerscheid, D. van Oosten, P. J. H. Denteer, and H. T. C. Stoof, Phys. Rev. A 68, $043623(2003)$

[13] X. Lu, J. Li, and Y. Yu, Phys. Rev. A 73, 043607 (2006)

[14] V.N. Popov, Functional Integrals in Quantum Field Theory and Statistical Physics (Reidel, Dordrecht, 1983)

[15] S.L. Cornish, N. R. Claussen, J. L. Roberts, E. A. Cornell, and C. E. Wieman, Phys. Rev. Lett. 85, 1795 (2000)

[16] M. Linn and A.L. Fetter, Phys. Rev A 60, 4910 (1999)

[17] F. Dalfovo and S. Stringari, Phys. Rev. A 53, 2477 (1996)

\section{Appendix A}

For a Bose system with creation operators $a_{x}^{+}$and annihilation operators $a_{x}$ at a lattice site $x$, an appropriate definition of the condensate density is [3]

$$
n_{0}:=\lim _{x-x^{\prime} \rightarrow \infty}\left\langle a_{x}^{+} a_{x^{\prime}}\right\rangle .
$$

In our slave-boson representation, a creation process of a particle is associated to the product $b_{x}^{*} e_{x}$ and an annihilation process to $e_{x}^{*} b_{x}$, thus

$$
n_{0}=\lim _{x-x^{\prime} \rightarrow \infty}\left\langle b_{x}^{*} e_{x} e_{x^{\prime}}^{*} b_{x^{\prime}}\right\rangle .
$$

Here, the expectation value is defined with respect the functional integral (5) by

$$
\langle\cdots\rangle=\frac{1}{Z} \int \cdots \exp [\ldots] \prod_{x} \delta\left(\left|b_{x}\right|^{2}+\left|e_{x}\right|^{2}-1\right) \mathrm{d} b_{x} \mathrm{~d} b_{x}^{*} \mathrm{~d} e_{x} \mathrm{~d} e_{x}^{*} \mathrm{~d} \Phi_{x} \mathrm{~d} \Phi_{x}^{*} \mathrm{~d} \varphi_{x} .
$$

We are interested in the connection between the correlation function $\left\langle\Phi_{x} \Phi_{x^{\prime}}^{*}\right\rangle$ and the condensate density. For this purpose we integrate out the field $\Phi$ to transform the correlation function of the field $\Phi$ back to a correlation function of the fields $b$ and $e$. Therefore, we perform the integration

$\beta^{\prime 2} \int \Phi_{y} \Phi_{y^{\prime}}^{*} \exp \left[\beta^{\prime} \sum_{x, x^{\prime}} \Phi_{x}\left(\mathbf{1}-\hat{t}^{\prime}\right)^{-1} \Phi_{x^{\prime}}^{*}+\beta^{\prime} \sum_{x} \Phi_{x} b_{x}^{*} e_{x}+\beta^{\prime} \sum_{x} \Phi_{x}^{*} e_{x}^{*} b_{x}\right] \prod_{x} \mathrm{~d} \Phi_{x} \mathrm{~d} \Phi_{x}^{*}=$ 
A renormalized Gross-Pitaevskii Theory and vortices in a strongly interacting Bose gas 11
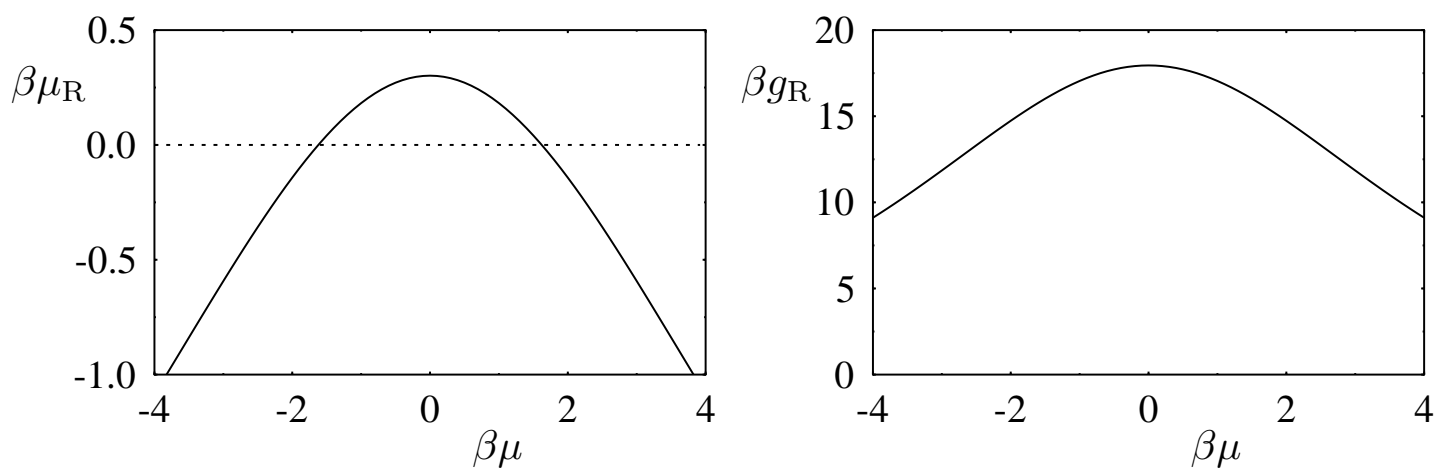

Figure 1. Coefficients $\mu_{\mathrm{R}}$ and $g_{\mathrm{R}}$ of the RGP theory plotted against the chemical potential $\mu$ for the tunneling rate $\alpha J=k_{\mathrm{B}} T$. Both functions are symmetric in $\mu$. A BEC exists if $\mu_{\mathrm{R}}>0$. The two points where $\mu_{\mathrm{R}}=0$ mark the phase transition between the non-condensate and the BEC. The coefficient $g_{\mathrm{R}}$ ist always positive.

$\frac{\partial}{\partial\left(b_{y}^{*} e_{y}\right)} \frac{\partial}{\partial\left(b_{y^{\prime}} e_{y^{\prime}}^{*}\right)} \int \exp \left[\beta^{\prime} \sum_{x, x^{\prime}} \Phi_{x}\left(\mathbf{1}-\hat{t}^{\prime}\right)^{-1} \Phi_{x^{\prime}}^{*}+\beta^{\prime} \sum_{x} \Phi_{x} b_{x}^{*} e_{x}+\beta^{\prime} \sum_{x} \Phi_{x}^{*} e_{x}^{*} b_{x}\right] \prod_{x} \mathrm{~d} \Phi_{x} \mathrm{~d} \Phi_{x}^{*}=$ $\frac{\partial}{\partial\left(b_{y}^{*} e_{y}\right)} \frac{\partial}{\partial\left(b_{y^{\prime}} e_{y^{\prime}}^{*}\right)} \operatorname{det} \beta^{\prime}\left(\mathbf{1}-\hat{t}^{\prime}\right) \exp \left[\beta^{\prime} \sum_{x, x^{\prime}} b_{x}^{*} e_{x}\left(\mathbf{1}-\hat{t}^{\prime}\right) e_{x^{\prime}}^{*} b_{x^{\prime}}\right]=$

$\beta^{\prime 2} \operatorname{det}\left(\mathbf{1}-\hat{t}^{\prime}\right)\left[\left(\mathbf{1}-\hat{t}^{\prime}\right)_{y, y^{\prime}}+\beta^{\prime} \sum_{x, x^{\prime}} b_{x}^{*} e_{x} e_{x^{\prime}}^{*} b_{x^{\prime}}\left(\mathbf{1}-\hat{t}^{\prime}\right)_{y^{\prime}, x^{\prime}}\right] \exp \left[\beta^{\prime} \sum_{x, x^{\prime}} b_{x}^{*} e_{x}\left(\mathbf{1}-\hat{t}^{\prime}\right)_{x, x^{\prime}} e_{x^{\prime}}^{*} b_{x^{\prime}}\right]$

Since we are interested in the limit $y-y^{\prime} \rightarrow \infty$, and the matrix $\hat{t}_{y, y^{\prime}}^{\prime}$ includes nearestneighbour hopping only, the term $\left(\mathbf{1}-\hat{t}^{\prime}\right)_{y, y^{\prime}}$ does not contribute. This yields for far distant lattice points $y, y^{\prime}$ the expression

$$
\left\langle\Phi_{y} \Phi_{y^{\prime}}^{*}\right\rangle=\sum_{x, x^{\prime}}\left\langle b_{x}^{*} e_{x} e_{x^{\prime}}^{*} b_{x^{\prime}}\right\rangle\left(\mathbf{1}-\hat{t}^{\prime}\right)_{x, y}\left(1-\hat{t}^{\prime}\right)_{y^{\prime}, x^{\prime}} .
$$

In this sum, only those terms contribute, where $x, y$ as well as $x^{\prime}, y^{\prime}$ are nearest neighbours. In the limit $y-y^{\prime} \rightarrow \infty$ we can assume $\left\langle b_{x}^{*} e_{x} e_{x^{\prime}}^{*} b_{x^{\prime}}\right\rangle=\left\langle b_{y}^{*} e_{y} e_{y^{\prime}}^{*} b_{y^{\prime}}\right\rangle$. Thus we can use

$$
\sum_{x}\left(\mathbf{1}-\hat{t}^{\prime}\right)_{x, y}=\sum_{x^{\prime}}\left(\mathbf{1}-\hat{t}^{\prime}\right)_{y^{\prime}, x^{\prime}}=(1+1 / \alpha)^{2} .
$$

We get

$$
\lim _{y-y^{\prime} \rightarrow \infty}\left\langle\Phi_{y} \Phi_{y^{\prime}}^{*}\right\rangle \approx(1+1 / \alpha)^{2} \lim _{y-y^{\prime} \rightarrow \infty}\left\langle b_{y}^{*} e_{y} e_{y^{\prime}}^{*} b_{y^{\prime}}\right\rangle
$$

and therefore

$$
n_{0} \approx \frac{1}{(1+1 / \alpha)^{2}} \lim _{y-y^{\prime} \rightarrow \infty}\left\langle\Phi_{y} \Phi_{y^{\prime}}^{*}\right\rangle .
$$

On the mean-field level, this justifies our identification of the condensate denity in Eq. (9). 


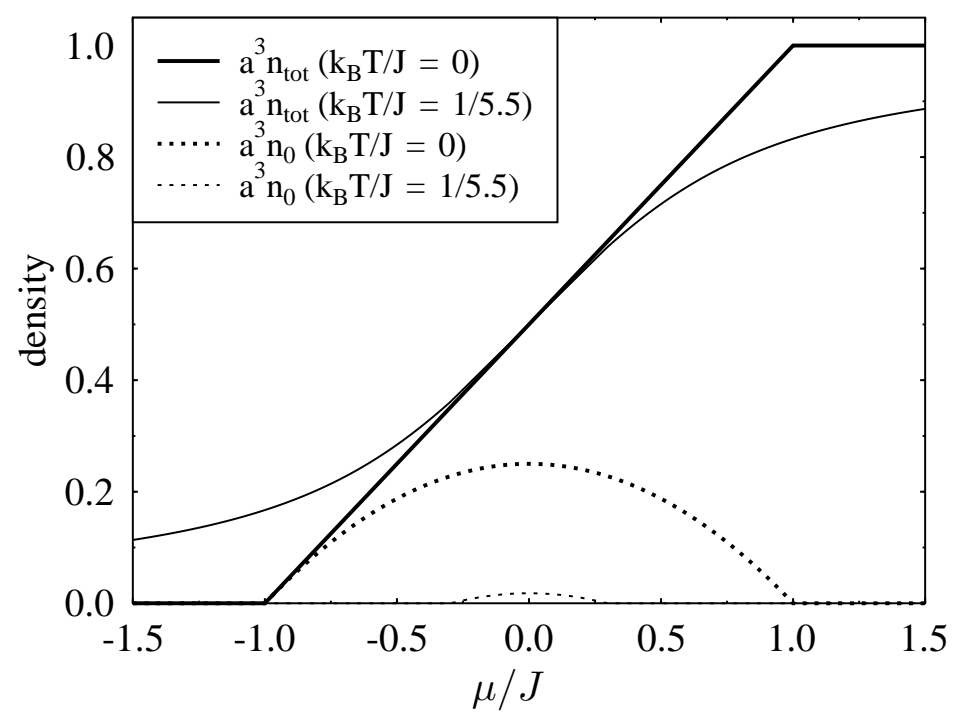

Figure 2. Condensate density $n_{0}$ and total density $n_{\text {tot }}$ of a translational invariant system, plotted against chemical potential for zero-temperature (thick lines) and finite temperature (thin lines).

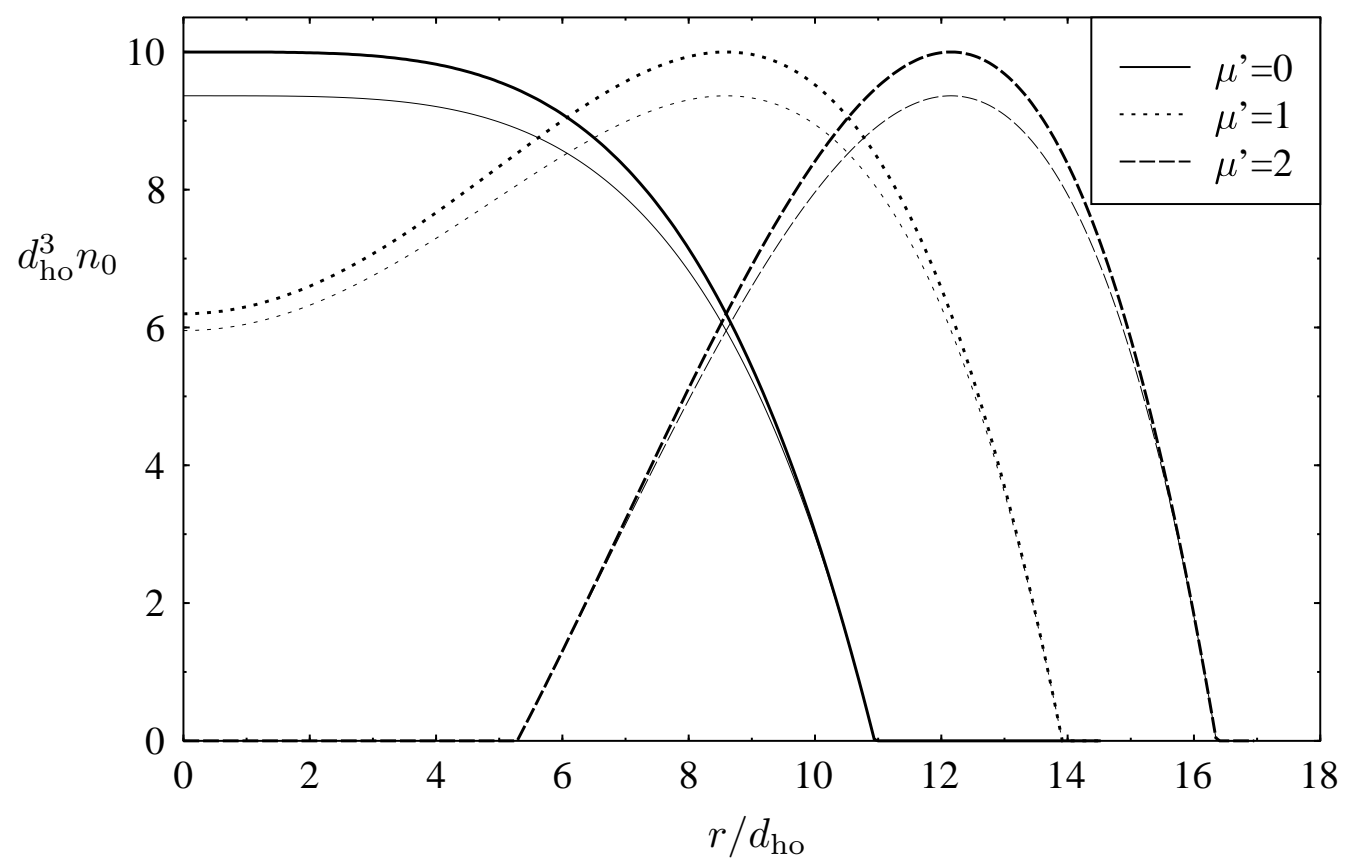

Figure 3. Condensate density $n_{0}=|\Phi|^{2} / a^{3}(1+1 / \alpha)^{2}$ of a vortex-free condensate in a spherical trap with the numerical parameters given in Eq. (23) and different values of the chemical potential $\mu^{\prime}$ calculated from the full slave-boson mean-field Eq. (24) (thick lines) and within RGP approximation (25) (thin lines). 


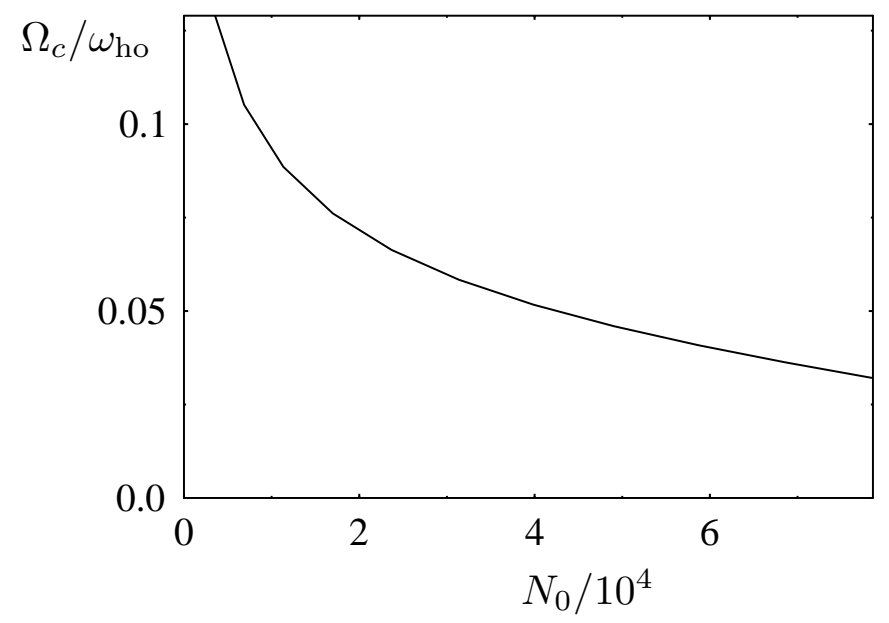

Figure 4. Critical angular velocity plotted against the total number of particles in the condensate.

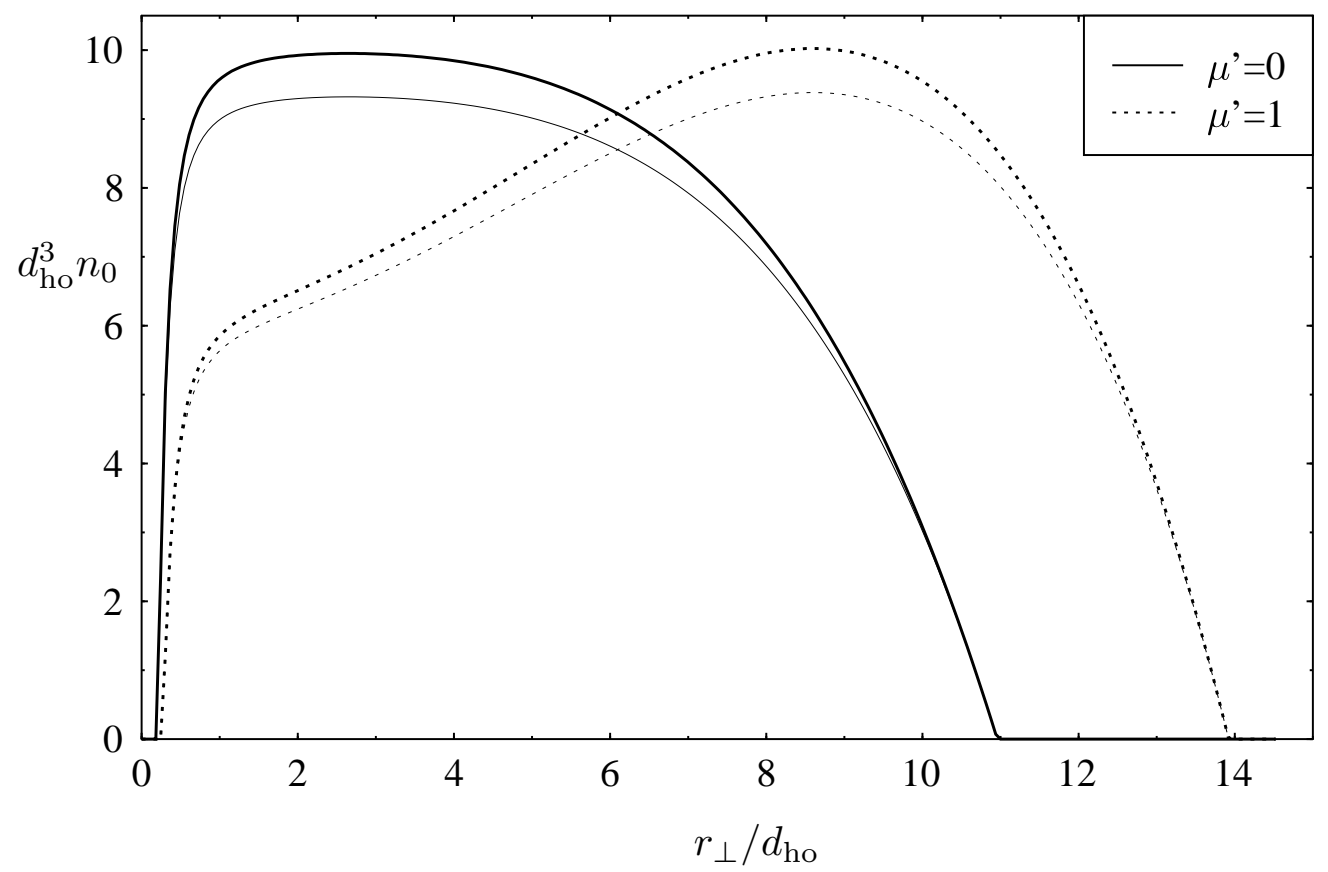

Figure 5. Condensate density of a condensate with a single vortex, with same parameters as in Fig. 3. calculated from the full mean-field Eq. (26) (thick lines) and within RGP approximation (27) (thin lines). The rotating frequencies of the trap were chosen to be close to the critical angular frequency $\Omega_{\mathrm{c}}$. 